\title{
Kinetic Energy Induced Smoothening and Delay of Epitaxial Breakdown in Pulsed-laser Deposition
}

\section{Citation}

Shin, Byungha and Michael J. Aziz. 2007. Kinetic energy induced smoothening and delay of epitaxial breakdown in pulsed laser deposition. Physical Review B 76(8): 085431.

\section{Published Version}

http://dx.doi.org/10.1103/PhysRevB.76.085431

\section{Permanent link}

http://nrs.harvard.edu/urn-3:HUL.InstRepos:2795319

\section{Terms of Use}

This article was downloaded from Harvard University's DASH repository, and is made available under the terms and conditions applicable to Other Posted Material, as set forth at http:// nrs.harvard.edu/urn-3:HUL.InstRepos:dash.current.terms-of-use\#LAA

\section{Share Your Story}

The Harvard community has made this article openly available.

Please share how this access benefits you. Submit a story.

Accessibility 


\title{
Kinetic energy induced smoothening and delay of epitaxial breakdown in pulsed laser deposition
}

\author{
Byungha Shin and Michael J. Aziz* \\ Harvard School of Engineering and Applied Sciences, Cambridge, MA 02138
}

(Version of 4/1/2007)

\begin{abstract}
We have isolated the effect of kinetic energy of depositing species during pulsed laser deposition (PLD) on surface morphology evolution of Ge(001) homoepitaxy at low temperature $\left(100^{\circ} \mathrm{C}\right)$. Using a dual molecular beam epitaxy (MBE)-PLD chamber, we compare morphology evolution from three different growth methods under identical experimental conditions except for the differing nature of the depositing flux: (a) PLD with average kinetic energy $300 \mathrm{eV}$ (PLD-KE); (b) PLD with suppressed kinetic energy comparable to thermal evaporation energy (PLD-TH); and (c) MBE. The thicknesses at which epitaxial breakdown occurs are ranked in the order PLD-KE > MBE > PLD-TH; additionally, the surface is smoother in PLD-KE than in MBE. The surface roughness of the films grown by PLD-TH cannot be compared due to the early epitaxial breakdown. These results convincingly demonstrate that the enhancement of epitaxial growth - the reduction in roughness and the delay of epitaxial breakdown - are due to the high kinetic energy of depositing species in PLD.

PACS code: 81.15.Fg; 68.55.-a; 61.14.Hg; 81.15.Hi
\end{abstract}




\section{INTRODUCTION}

Pulsed laser deposition (PLD) has emerged as an important growth technique as it can offer far-from-the-equilibrium growth conditions not readily accessible by conventional thermal deposition techniques such as molecular beam epitaxy (MBE). ${ }^{1,2}$ Two major fundamental differences are generally believed to distinguish PLD and MBE. In PLD, deposition occurs in short pulses resulting in a high instantaneous flux typically several orders of magnitude larger than the steady-state flux in MBE, although the timeaveraged deposition fluxes can be similar. Additionally, the kinetic energy of depositing species in PLD can be as large as a few hundred $\mathrm{eV}$, in contrast to the thermal energy of $<1 \mathrm{eV}$ in MBE. ${ }^{2,3}$ In order to understand PLD growth and its relationship to MBE, it is essential to learn the relative roles of the pulsed and energetic nature of the PLD flux in determining the growing structure and morphology.

Extensive studies over the past two decades have led to a good understanding of kinetic processes in MBE. Based on this understanding, manipulated processes, such as modulated flux ${ }^{4,5}$ or ion-beam assisted growth, ${ }^{6-8}$ have been met with some success in obtaining a desired surface morphology. These manipulated processes bear some similarities to PLD. Therefore, the same reasoning to explain the improvements in epitaxial growth by the manipulated processes is worthy of consideration for PLD as well. For example, flux modulation has been shown to enhance layer-by-layer growth due to an increased island density and a concomitant decreased average island size formed during the period of high deposition rate., ${ }^{5,9}$ Correspondingly, layer-by-layer growth might be improved by the high-flux bursts in PLD even without the energetic characteristic of depositing species. In contrast to this expectation, kinetic Monte-Carlo (KMC) 
simulations ${ }^{10,11}$ have shown that PLD without high kinetic energy (referred to as pulsed thermal deposition in general or PLD-TH in this article) yields a greater surface roughness than MBE. In their KMC studies, Hinnemann et al. ${ }^{11}$ suggested that the improved layer-by-layer growth by PLD over MBE should be attributed to the energetic effect. Experimentally, PLD has been demonstrated to produce smoother films in growing metal $^{12}$ or semiconductor ${ }^{3}$ materials than a thermal deposition technique under otherwise identical growth conditions. However, the relative contributions of the energetic and the pulsed natures of PLD in improving the smoothness remain undetermined.

Previously we performed a comparative study of morphology evolution of $\mathrm{Ge}(001)$ homoepitaxy at low temperatures by PLD and MBE using a dual MBE-PLD ultrahigh vacuum (UHV) chamber that provides identical growth conditions (e.g., substrate temperature calibration, background gas composition, substrate surface preparation) except for the different nature of the deposition flux. ${ }^{3}$ We found that in $\mathrm{Ge}(001)$ homoepitaxy at $150{ }^{\circ} \mathrm{C}$, PLD yields films as smooth as or smoother than MBE, depending on the kinetic energy of PLD. It was also shown that epitaxial breakdown, where epitaxial growth is no longer sustained and the growing phase becomes amorphous, is considerably postponed in the case of PLD. We argued that these differences between PLD and MBE are due mainly to the energetic effect of PLD. Here we report the first experimental results definitively isolating the role of kinetic energy in PLD. We compare the growth morphology in $\mathrm{Ge}(001)$ homoepitaxy at $100{ }^{\circ} \mathrm{C}$ by PLD with high kinetic energy (referred to as PLD-KE in this article), PLD-TH, and MBE. We find that PLD-KE produces smoother films compared to MBE. The epitaxial breakdown is delayed in PLD- 
KE, but in PLD-TH it occurs earlier than MBE. We also address the relaxation kinetics during the growth interruptions between laser pulses by varying the repetition rate in PLD.

\section{EXPERIMENTS}

Both MBE and PLD growth have been conducted in a dual MBE-PLD UHV chamber with a base pressure of mid $10^{-11}$ Torr. The details of the sample preparation and growth by MBE and PLD-KE have been reported elsewhere. ${ }^{3}$ For PLD-TH, we deliberately introduce high purity He gas (99.999\%) into the chamber prior to PLD-TH growth. With a target-substrate separation of $6 \mathrm{~cm}$ approximately 0.3 Torr of He gas is sufficient to dissipate the kinetic energy of the plume to less than $0.1 \mathrm{eV}$ as measured by an ion probe residing on the sample holder. The average kinetic energy of PLD-KE is $300 \mathrm{eV} .{ }^{13}$ The kinetic energy distributions of PLD-KE and PLD-TH are shown in Fig. 1(a) and 1(b), respectively. Because trace contamination can significantly degrade the crystalline quality of $\mathrm{Ge}(001)$ films, we take extreme care to improve the purity of the He gas. A Ti getter operating at $\sim 200{ }^{\circ} \mathrm{C}$ is used to eliminate impurities from the inlet gas stream and a portion of the stainless steel tubing containing the gas flow both upstream and downstream of the Ti getter is immersed in liquid nitrogen to trap any moisture. With these precautions, no noticeable change in the reflection high energy electron diffraction (RHEED) pattern is detected over several hours of exposure to He gas, indicating that the surface is impervious to the gas introduction. For RHEED intensity measurements, a beam incidence angle of $0.64^{\circ}$ and azimuth $7^{\circ}$ off $<110>$ are chosen to prevent interference of the specular spot by the Kikuchi features. ${ }^{14}$ The film thickness is 
determined by Rutherford Backscattering Spectrometry (RBS) on a companion sample of Ge grown simultaneously on Si.

\section{RESULTS AND DISCUSSION}

In Figs. 2(a) - 2(c) we show atomic force microscopy (AFM) topographs of the surface morphology of $\sim 30 \mathrm{~nm}$ films grown at $100{ }^{\circ} \mathrm{C}$ by MBE, PLD-KE, and PLD-TH. The morphology of low-temperature $\mathrm{Ge}(001)$ homoepitaxy is known to evolve from an array of irregularly shaped islands to well-defined pyramidal growth mounds. ${ }^{3,15}$ The surface morphology shown in Figs. 2(a) and 2(b) are in mid transition to the pyramidal mounds morphology. In the case of PLD-TH, shown in Fig. 2(c), the surface consists of irregularly shaped smaller islands and large surface features (LSFs) marked by the arrows near the bottom of Fig. 2(c), of 3-5 nm height. The areal density of the LSFs is roughly $10 \%$; figure 2(c) is from one of a few areas with a relatively low density of such features. Figure 2(d) shows a RHEED pattern taken at the surface of Fig. 2(c). It contains a set of transmission diffraction spots and amorphous rings indicating the occurrence of epitaxial breakdown at this thickness. In contrast, the films grown by MBE and PLD-KE well beyond this thickness are still fully epitaxial as confirmed by RHEED patterns not shown.

In films grown by PLD are often observed particulates, which are believed to be directly ejected from a roughened target. ${ }^{1}$ Particulates are much larger than the LSFs typically on the order of a micron. We observe micron-sized particulates as well as the LSFs in PLD-TH. Furthermore, we see micron-sized particulates but no LSFs in other PLD-TH films with thickness $<27 \mathrm{~nm}$. Hence we suggest that the LSFs are the amorphous phase accounting for the amorphous rings in Fig. 2(d). 
For quantification of the evolution of the surface morphology, a plot of rootmean-square (rms) roughness vs. film thickness for PLD-TH, PLD-KE, and MBE is shown in Fig. 3. In the case of PLD-KE, we have varied the repetition rate from 5 to 20 $\mathrm{Hz}$ while keeping the instantaneous flux the same (thereby varying the time-averaged deposition rate) to observe the influence of relaxation during the interruptions between deposition pulses in PLD. One can see that PLD-KE produces smoother films than MBE. Due to the epitaxial breakdown occurring at $\sim 27 \mathrm{~nm}$ in PLD-TH, we cannot compare the roughness in PLD-TH to the other methods.

Most noteworthy is a different onset of epitaxial breakdown for different growth methods as indicated by the dotted vertical arrows in Fig. 3. For PLD-KE, all the data presented in the figure are free of epitaxial breakdown. Different epitaxial thicknesses between PLD-TH $(27 \mathrm{~nm})$ and PLD-KE (> $270 \mathrm{~nm})$ directly suggest that the kinetic energy of depositing species is the most crucial factor in determining the epitaxial thickness. However, before drawing conclusions about the role of the kinetic energy, we must address three additional differences among our growth conditions — other than the pulsed and energetic nature of the deposition flux — that might affect epitaxial breakdown.

First, in PLD-TH, the average deposition rate is an order of magnitude smaller, $\sim 0.003 \mathrm{~nm} / \mathrm{sec}$, than in the other cases (MBE and PLD-KE with 20Hz), $\sim 0.03 \mathrm{~nm} / \mathrm{sec}$ presumably due to the scattering-induced divergence of the plume. It is generally believed that decreasing the deposition rate should help to delay epitaxial breakdown as it permits a longer time for deposited species to travel to "correct" sites. This is the opposite of the effect we observe. 
Second, RHEED is turned off during PLD-TH whereas in MBE and PLD-KE the electron beam is incident on the growing surface continuously for in-situ monitoring of crystalline quality. Because the impingement of high energy electrons can generate surface defects such as vacancies. ${ }^{16}$ it is possible that the RHEED beam during growth alters the surface structure by creating surface defects. However, the estimated defect generation rate is extremely low compared to the deposition rate owing to a very small flux of the RHEED beam on the sample. ${ }^{17}$ To support this argument we performed the following control experiments. We performed two MBE growths - one with a low deposition rate comparable to that of PLD-TH and the other with the RHEED beam off during deposition - and compared the epitaxial thickness with that of "normal" MBE (higher deposition rate and RHHED on). It turns out that the epitaxial thickness in both control experiments is $\sim 80 \mathrm{~nm}$, which falls within the range of epitaxial thickness by "normal" MBE, $70 \mathrm{~nm}$ (determined by RBS channeling) - $80 \mathrm{~nm}$ (determined by the RHEED pattern). We conclude that an order of magnitude difference in deposition rate and the incident RHEED beam during growth hardly affect the breakdown.

Third, in addition to particulate formation, in PLD in an ambient gas the formation of nanometer-sized clusters has been reported. ${ }^{18,19}$ The ambient gas opposes rapid expansion of the PLD plume and confines it to a smaller region. As a result the concentration of the ablated species increases leading to a higher probability of cluster nucleation. Additionally, these nanoclusters are stabilized via collisional "cooling” by transfer of the latent heat of condensation to ambient gas molecules and stable nanoclusters can be transported to a substrate. ${ }^{20}$ It is conceivable that such nanoclusters form in our PLD-TH experiments and they may catalyze the transition to amorphous 
growth, thereby explaining the earlier epitaxial breakdown in PLD-TH. During ablation of Si under He gas, the typical range of He background gas pressure to form nanoclusters is reported to be $0.2-10$ Torr. ${ }^{18}$ Considering the difference between the mass of Si and $\mathrm{Ge}$, it is reasonable to assume that a higher pressure of He would be needed for the nanocluster formation in the case of Ge. Therefore, our He pressure of 0.3 Torr during PLD-TH appears to be a very marginal condition to form nanoclusters. Furthermore, nanoclusters arriving on a substrate are expected to have different crystallographic orientations from that of the substrate due to the lack of kinetic energy to rearrange themselves on the surface and should consequently give rise to additional peaks other than those from the substrate in x-ray diffraction (XRD) measurements. ${ }^{19}$ XRD $\theta-2 \theta$ scans on our samples reveal no additional peaks observed from PLD-TH when compared to PLD-KE and MBE. Furthermore, RHEED patterns collected from PLD-TH films exhibit no signature of diffraction from other surface orientations except that from (001) plane. So it is unlikely that nanoclusters are on the surface under our PLD-TH conditions.

From the evidence above we conclude that the delay of epitaxial breakdown in PLD-KE is predominantly due to the high kinetic energy of depositing species. Our conclusion is further supported by a set of data obtained from growth at $150{ }^{\circ} \mathrm{C}$, which demonstrates that epitaxial breakdown is delayed in PLD-KE but it occurs earlier in PLD-TH when compared to MBE, just like in the case of $100{ }^{\circ} \mathrm{C}$. Table I summarizes the epitaxial thickness of PLD-KE, PLD-TH, and MBE at both $100{ }^{\circ} \mathrm{C}$ and $150{ }^{\circ} \mathrm{C}$. A generally accepted mechanism for epitaxial breakdown is that defects such as stacking faults or twins nucleate at deep trenches between mounds developed due to kinetic roughening, and the accumulation of these defects results in the transition to the growth 
of the amorphous phase. ${ }^{21}$ It has been proposed that incoming atoms with high kinetic energy may generate mobile surface defects that then migrate into the troughs between the mounds preventing the formation of the deep trenches. ${ }^{3,6}$ Also the impact of energetic species has been proposed to locally deliver momentum to atoms non-epitaxially deposited or overhung within the trenches, pushing them to epitaxial sites near the bottom of the trenches; this collision-induced filling makes it more difficult for the defects to nucleate, thereby delaying epitaxial breakdown. ${ }^{7}$

Finally we consider the relaxation kinetics during PLD-KE. As shown in Fig. 3, the roughness evolution of PLD-KE appears to follow a single trend irrespective of the repetition rate. In other words, varying the time available for relaxation between deposition pulses from $\sim 1 / 20$ sec to $\sim 1 / 5$ sec seems to have no systematic effect on the surface roughness. To gain more insight to this matter, we focus on the early stage of PLD-KE by monitoring the intensity variations of the RHEED specular spot, as shown in Fig. 4. Two different repetition rates $-1 \mathrm{~Hz}$ and $2 \mathrm{~Hz}$ - are used to offer different relaxation times. Note that the time axes in Figs. 4(a) and 4(b) are adjusted such that the intensity minima and maxima are aligned at the same positions on the abscissa but the time axes are not related by exactly a factor of two due to the decreased deposition rate from target aging for the run with $2 \mathrm{~Hz}$. Figure 4(a) demonstrates that within the experimental fluctuations the curves are almost identical to each other. Furthermore, as shown in Fig. 4(b), the intensity between pulses remains nearly constant within the noise level of RHEED signal. In PLD, intensity modulations of specularly reflected RHEED ${ }^{22}$ and $\mathrm{XRD}^{23,24}$ spots similar to our results, have been reported. Some of those measurements exhibit intensity recovery between pulses; this has been attributed to 
interlayer transport ${ }^{23,24}$ or reduction of adatom density ${ }^{22}$. Our observation of an insignificant intensity change during the growth interruptions between pulses following each abrupt change during a burst of deposition suggests that the surface is effectively frozen during the inter-pulse periods. However, we believe that very fast athermal transport mechanisms such as adatom insertion ${ }^{25}$, island breakup ${ }^{23}$, or transient enhanced mobility of small-sized clusters on the surface ${ }^{26}$ may still operate during or immediately following a deposition pulse in our PLD-KE. Any structural rearrangement on the surface by athermal mechanisms, as well as by thermal transport if it also operates, ${ }^{27}$ is completed within $\sim 1 / 30$ second - which is the sampling rate of our RHEED intensity measurements — after a laser pulse. Therefore, varying the repetition rate from 5 to 20 $\mathrm{Hz}$ should have a negligible effect on the surface morphology, which is consistent with the observations in Fig. 3.

In our argument given in the previous paragraph in explaining a similar roughness evolution of PLD-KE with repetition rates varying from 5 to $20 \mathrm{~Hz}$, we implicitly assumed that there is no recovery between deposition pulses at all coverages. However, we should admit that our RHEED intensity variation spectra between pulses tend to become noisier near full coverage than at lower coverages such as shown in Fig. 4(b), so we cannot be certain that there is absolutely no recovery near full coverage. It has been shown that sometimes ${ }^{24}$, but not always ${ }^{25}$, recovery between pulses is observable only near full coverage while there is no apparent recovery at lower coverages. However, the observation of the nearly identical intensity variations between $1 \mathrm{~Hz}$ and $2 \mathrm{~Hz}$ [shown in Fig. 4(a)] and the similar roughness evolution regardless of the repetition rate $(5-20 \mathrm{~Hz}$, shown in Fig. 3) as well as the successful scaling of the epitaxial thickness with 
instantaneous growth rate, as discussed in the next paragraph, are consistent with our hypothesis that there is no recovery between pulses at all coverages.

The RHEED intensity measurements shown in Fig. 4 can also help us to understand the earlier epitaxial breakdown in PLD-TH. Because there is insignificant structural change between deposition pulses during PLD-KE, it is fair to assume that the same is true for PLD-TH. Additionally, because the fast athermal transport mechanisms are absent in PLD-TH we can consider PLD-TH to be the kinetic equivalent of "MBE" with a very high deposition rate: all processes are simply "turned off" during the interval between pulses. Assuming a pulse duration of $10 \mu \mathrm{s}$, the instantaneous deposition rate in our PLD-TH runs is $\sim 15 \mathrm{~nm} / \mathrm{sec}$, which is 500 times that of our MBE runs. In low temperature $\mathrm{Si}(001)$ homoepitaxy by $\mathrm{MBE}^{28}$, the epitaxial thickness $h_{e p i}$ at $260{ }^{\circ} \mathrm{C}$ exhibits a logarithmic or a weak power-law dependence on the deposition rate such as $h_{e p i}$ $\sim F^{-n}$, where $F$ is the deposition rate and the power $n$ is 0.22 . Assuming a power-law dependence were also to relate our measurements of $h_{e p i}$ in MBE and PLD-TH (with PLD-TH treated as MBE with a deposition rate 500 times as fast) yields a power of $n=$ $0.16-0.18$ and 0.20 for $100{ }^{\circ} \mathrm{C}$ and $150{ }^{\circ} \mathrm{C}$, respectively, which is close to 0.22 from low temperature $\mathrm{Si}(001)$ homoepitaxy. This supports our speculation that PLD-TH behaves like MBE. We conclude that the energetic nature of the flux in PLD is the most prominent feature that distinguishes PLD from MBE in low temperature Ge(001) homoepitaxy.

\section{SUMMARY}


In summary, we have isolated the effect of the kinetic energy of depositing species during PLD by comparing the surface morphology of PLD-TH, PLD-KE, and MBE under identical experimental conditions except for the differing nature of the depositing flux. We find that the epitaxial thicknesses are ranked in the order of PLD-KE $>$ MBE $>$ PLD-TH and the surface roughness in PLD-KE is much less than in MBE. The dependence of roughness on laser pulse repetition rate is negligible and is consistent with the negligible relaxation observed between pulses by time-resolved RHEED. Our results convincingly demonstrate that the enhancement of epitaxial growth - the reduction in roughness and the delay of epitaxial breakdown - are due to the high kinetic energy of depositing species in PLD.

\section{ACKNOWLEDGEMENTS}

We acknowledge J. P. Leonard for technical assistance and D. G. Cahill for guidance in cleaning Ge This research was supported by NSF DMR-0306997. 
*email: maziz at harvard dot edu

\section{REFERENCES}

${ }^{1}$ Pulsed Laser Deposition of Thin Films, edited by D. B. Chrisey and G.K. Hubler. (John Wiley, New York, 1994).

${ }^{2}$ P. R. Willmott and J. R. Huber, Reviews of Modern Physics 72, 315 (2000).

${ }^{3}$ B. Shin, J. P. Leonard, J. W. McCamy, and M. J. Aziz, Appl. Phys. Lett. 87, 181916 (2005).

${ }^{4}$ V. A. Markov, O. P. Pchelyakov, L. V. Sokolov, S. I. Stenin, and S. Stoyanov, Surf. Sci. 250, 229 (1991); S. Schinzer, M. Sokolowski, M. Biehl, and W. Kinzel, Phys. Rev. B 60, 2893 (1999).

${ }^{5}$ M.I. Larsson, W.-X. Ni, and G.V. Hansson, J. Appl. Phys. 78, 3792 (1995).

${ }^{6}$ E. Chason, P. Bedrossian, K.M. Horn, J.Y. Tsao, and S.T. Picraux, Appl. Phys. Lett. 57, 1793 (1990).

${ }^{7}$ D. L. Smith, C. C. Chen, G. B. Anderson, and S. B. Hagstrom, Appl. Phys. Lett. 62, 570 (1993); N. E. Lee, G. Xue, and J. E. Greene, J. Appl. Phys. 80, 769 (1996).

${ }^{8}$ M. Kalff, M. Breeman, M. Morgenstern, T. Michely, and G. Comsa, Appl. Phys. Lett. 70, 182 (1997).

${ }^{9}$ G. Rosenfeld, B. Poelsema, and G. Comsa, J. Cryst. Growth 151, 230 (1995).

${ }^{10}$ M.E. Taylor and H.A. Atwater, Appl. Surf. Sci. 127-129, 159 (1998).

${ }^{11}$ B. Hinnemann, H. Hinrichsen, and D. E. Wolf, Phys. Rev. E 67, 011602 (2003).

${ }^{12}$ P. Ohresser, J. Shen, J. Barthel, M. Zheng, C. V. Mohan, M. Klaua, and J. Kirschner, Phys. Rev. B 59, 3696 (1999). 
${ }^{13}$ Note that PLD with this range of kinetic energy is referred to as PLD-LE in Ref. 3.

${ }^{14}$ B. Shin, J. P. Leonard, J. W. McCamy, and M. J. Aziz, in press (2007).

15 J. E. Van Nostrand, S. J. Chey, and D. G. Cahill, Phys. Rev. B 57, 12536 (1998).

${ }^{16}$ B. Y. Han, K. Nakayama, and J. H. Weaver, Phys. Rev. B 60, 13846 (1999).

17 The flux on the sample in RHEED is $\sim 3 \times 10^{14} \mathrm{~cm}^{-2} \mathrm{sec}^{-1}$. Assuming a vacancy creation yield of $1 \times 10^{-4}$ per incident $15 \mathrm{keV}$ electron, compared with a yield reported in Ref. 16 of $1 \times 10^{-5}$ for $2 \mathrm{keV}$ electrons on $\mathrm{Si}(111)$, we estimate that $1 \times 10^{-5} \mathrm{ML}$ of vacancies are created per second while $0.2 \mathrm{ML}$ is deposited per second.

${ }^{18}$ D. B. Geohegan, A. A. Puretzky, G. Duscher, and S. J. Pennycook, Appl. Phys. Lett. 72, 2987 (1998); A. V. Kabashin, J. P. Sylvestre, S. Patskovsky, and M. Meunier, J. Appl. Phys. 91, 3248 (2002).

${ }^{19}$ X. Y. Chen, Y. F. Lu, Y. H. Wu, B. J. Cho, M. H. Liu, D. Y. Dai, and W. D. Song, J. Appl. Phys. 93, 6311 (2003).

${ }^{20}$ W. Marine, L. Patrone, B. Luk'yanchuk, and M. Sentis, Appl. Surf. Sci. 154, 345 (2000).

${ }^{21}$ K. A. Bratland, Y. L. Foo, Jant Soares, T. Spila, P. Desjardins, and J. E. Greene, Phys. Rev. B 67, 125322 (2003).

${ }^{22}$ D.H.A. Blank, G. Koster, G.A.J.H.M. Rijnders, E. van Setten, P. Slycke, and H. Rogalla, J. Cryst. Growth 211, 98 (2000).

${ }^{23}$ P. R. Willmott, R. Herger, C.M. Schlepütz, D. Martoccia, and B.D. Patterson, Phys. Rev. Lett. 96, 176102 (2006).

${ }^{24}$ J. Tischler, G. Eres, B. Larson, C. M. Rouleau, P. Zschack, and D. H. Lowndes, Phys. Rev. Lett. 96, 226104 (2006). 
${ }^{25}$ A. Fleet, D. Dale, Y. Suzuki, and J. D. Brock, Phys. Rev. Lett. 94 (2005).

${ }^{26}$ E. Vasco and J. L. Sacedon, Phys. Rev. Lett. 98, 036104 (2007).

${ }^{27}$ It was shown that in $\mathrm{SrTiO}_{3}$ homoepitaxy by PLD faster non-thermal transport dominates over slower thermal transport at $650^{\circ} \mathrm{C}$. See Ref. 24 .

${ }^{28}$ D.J. Eaglesham, J. Appl. Phys. 77, 3597 (1995). 


\section{FIGURE CAPTIONS}

Fig. 1. Kinetic energy distribution of (a) PLD-KE and (b) PLD-TH. Note the difference in scale of $\mathrm{x}$-axis between (a) and (b).

Fig. 2. (Color online) AFM images of films grown at $100^{\circ} \mathrm{C}$ by (a) MBE, (b) PLD-KE, and (c) PLD-TH, and (d) RHEED pattern taken from surface shown in (c). Scan size and vertical scale of (a) - (c) are $0.25 \times 0.25 \mu \mathrm{m}^{2}$ and $5 \mathrm{~nm}$, respectively. Thickness of films in (a) - (c) is shown in the left bottom corner of each image.

Fig. 3. Log-log plot of rms roughness vs film thickness. Vertical dotted lines at $27 \mathrm{~nm}$ and at $70 \mathrm{~nm}$ (determined by RBS channeling) - $80 \mathrm{~nm}$ (determined by RHEED pattern) indicate the epitaxial thickness of PLD-TH and MBE films, respectively, grown at $100^{\circ} \mathrm{C}$.

Fig. 4. (a) RHEED specular intensity variations during PLD-KE with $1 \mathrm{~Hz}$ (dashed line) and $2 \mathrm{~Hz}$ (solid line) of repetition rate while keeping instantaneous flux the same in both cases. Time axes are adjusted such that intensity minima and maxima for both cases are aligned at the same positions on abscissa. (b) First 20 seconds of RHEED intensity variation of PLD-KE with $1 \mathrm{~Hz}$ from (a). Modulations from individual laser pulses are visible. 
Fig. 1

B. Shin and M. J. Aziz
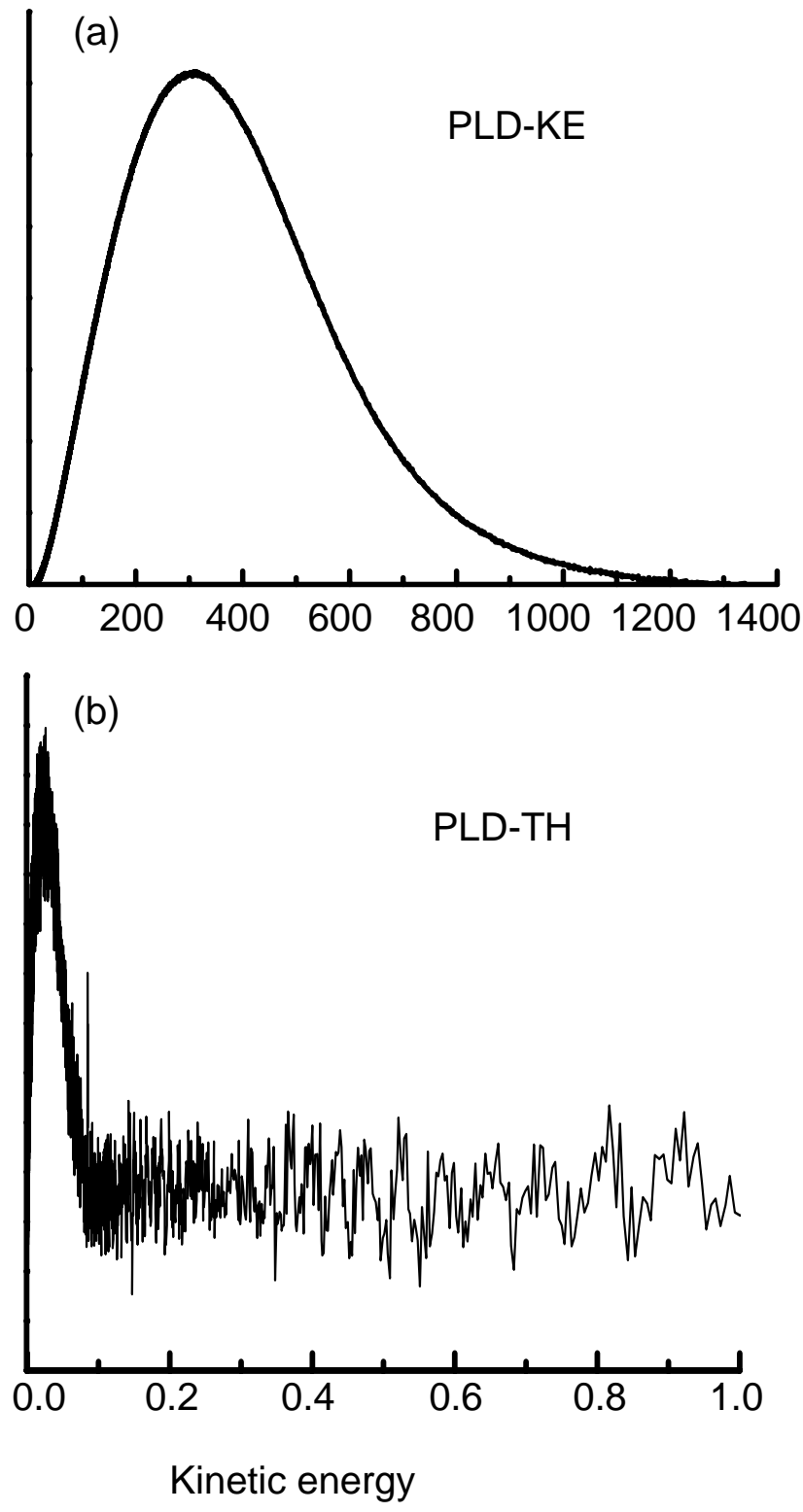

Fig.1. Kinetic energy distribution of (a) PLD-KE and (b) PLD-TH. Note the difference in scale of $\mathrm{x}$-axis between (a) and (b). 
Fig. 2

B. Shin and M. J. Aziz
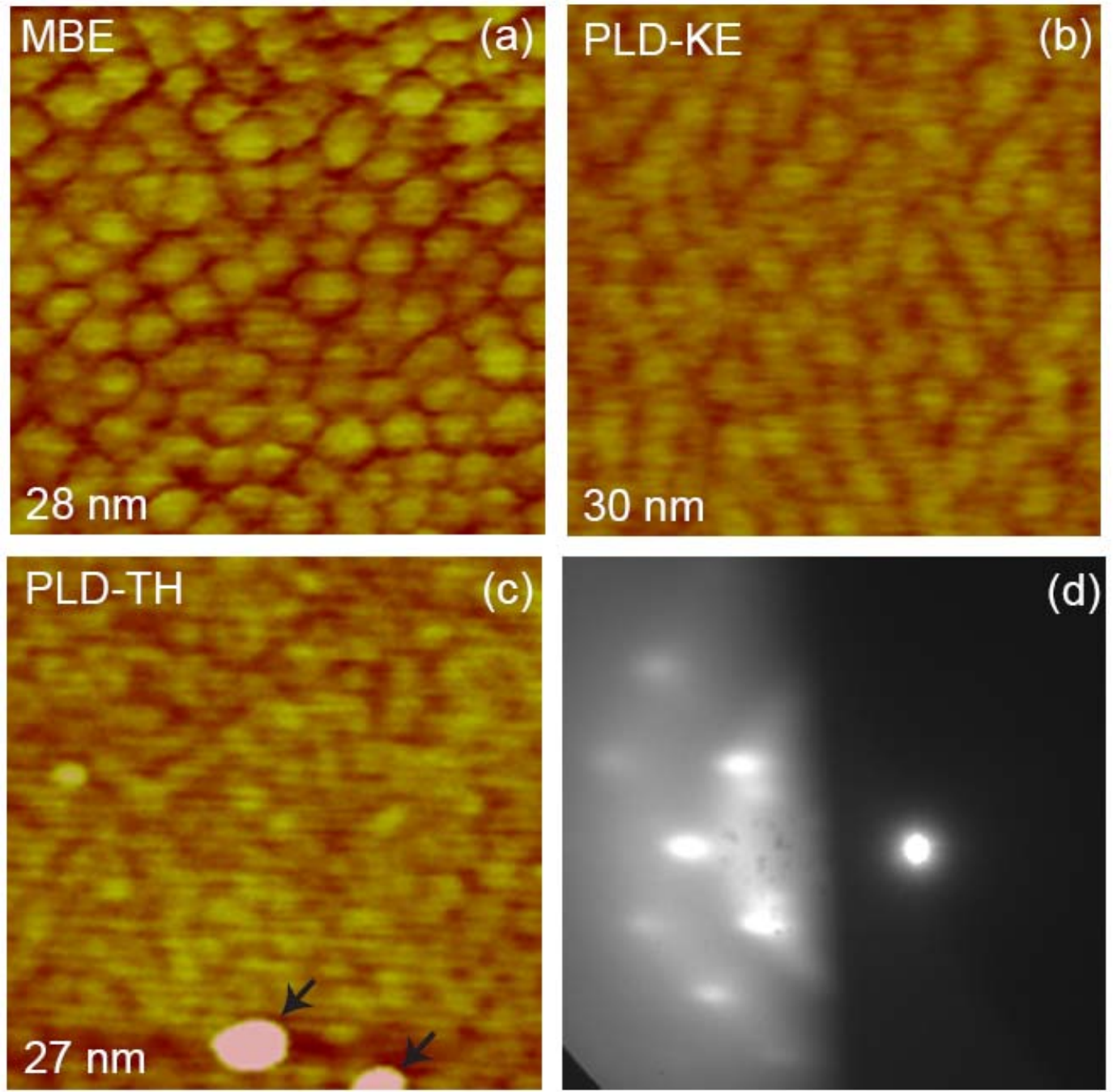

Fig. 2. (Color online) AFM images of films grown at $100{ }^{\circ} \mathrm{C}$ by (a) MBE, (b) PLD-KE, and (c) PLD-TH, and (d) RHEED pattern taken from surface shown in (c). Scan size and vertical scale of (a) - (c) are $0.25 \times 0.25 \mu \mathrm{m}^{2}$ and $5 \mathrm{~nm}$, respectively. Thickness of films in (a) - (c) is shown in the left bottom corner of each image. 
Fig. 3

B. Shin and M. J. Aziz

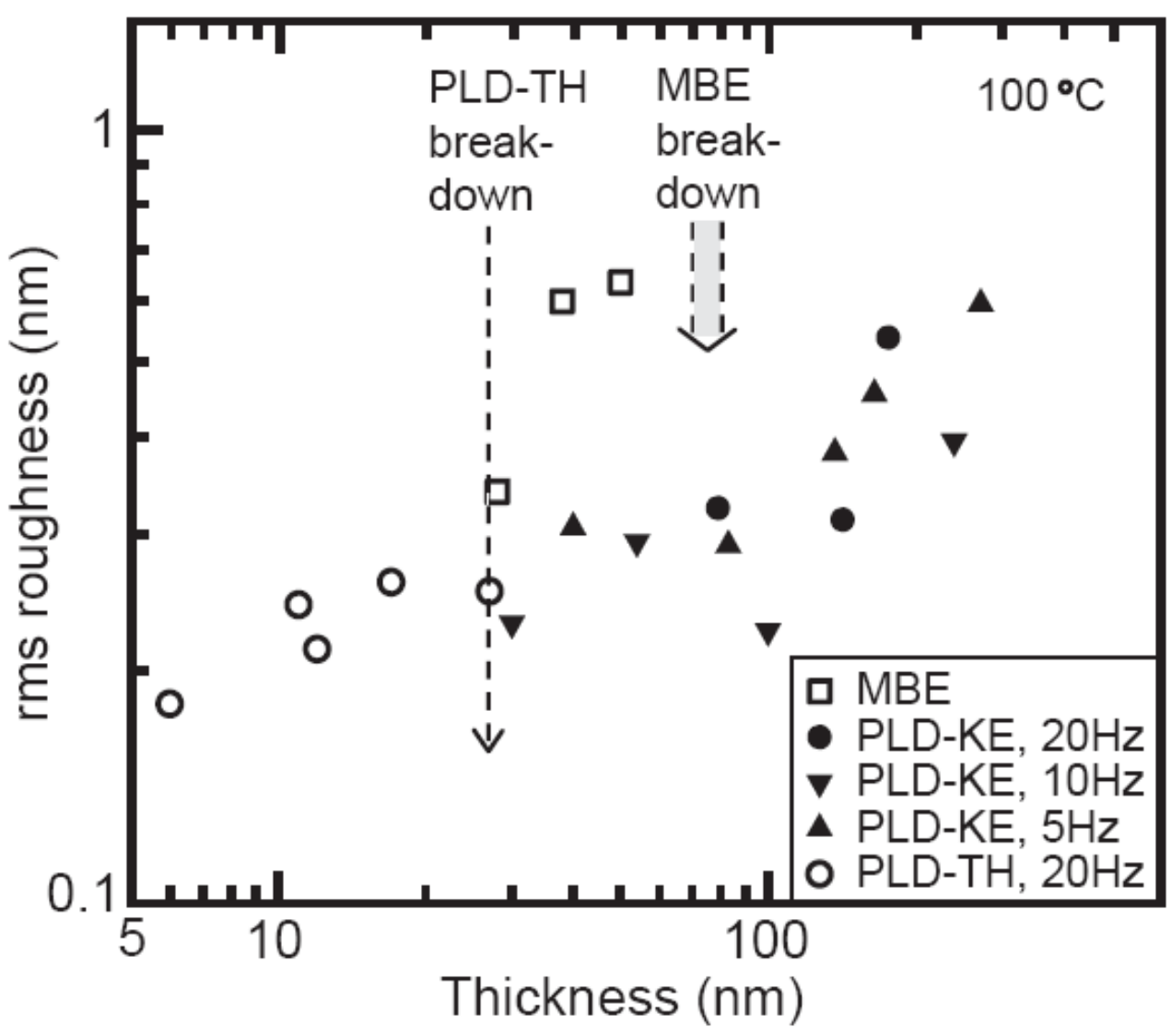

Fig. 3. Log-log plot of rms roughness vs film thickness. Vertical dotted lines at $27 \mathrm{~nm}$ and at $70 \mathrm{~nm}$ (determined by RBS channeling) - $80 \mathrm{~nm}$ (determined by RHEED pattern) indicate the epitaxial thickness of PLD-TH and MBE films, respectively, grown at $100^{\circ} \mathrm{C}$. 
Fig. 4

B. Shin and M. J. Aziz


Fig. 4. (a) RHEED specular intensity variations during PLD-KE with $1 \mathrm{~Hz}$ (dashed line) and $2 \mathrm{~Hz}$ (solid line) of repetition rate while keeping instantaneous flux the same in both cases. Time axes are adjusted such that intensity minima and maxima for both cases are aligned at the same positions on abscissa. (b) First 20 seconds of RHEED intensity variation of PLD-KE with $1 \mathrm{~Hz}$ from (a). Modulations from individual laser pulses are visible. 
Table I

\begin{tabular}{cccc} 
Growth Temperature & PLD-KE & PLD-TH & MBE \\
\hline \hline $100{ }^{\circ} \mathrm{C}$ & $>270 \mathrm{~nm}$ & $27 \mathrm{~nm}$ & $70-80 \mathrm{~nm}$ \\
$150{ }^{\circ} \mathrm{C}$ & $>410 \mathrm{~nm}$ & $60 \mathrm{~nm}$ & $210 \mathrm{~nm}$
\end{tabular}

Table I. Epitaxial thickness of PLD-KE, PLD-TH, and MBE at $100^{\circ} \mathrm{C}$ and $150{ }^{\circ} \mathrm{C}$. In the case of PLD-KE, the thickest samples $-270 \mathrm{~nm}$ at $100{ }^{\circ} \mathrm{C}$ and $410 \mathrm{~nm}$ at $150{ }^{\circ} \mathrm{C}$ - are still fully epitaxial. 